\title{
Response to crizotinib in a lung adenocarcinoma patient harboring a novel SLC34A2-ROSI fusion variant
}

This article was published in the following Dove Press journal:

OncoTargets and Therapy

21 August 2017

Number of times this article has been viewed

Zheng Zhao,' Zhangjun Song,' Xuwei Wang, ${ }^{3}$ Haifeng Sun,' Xiaomin Yang, ${ }^{2}$ Yong Yuan, ${ }^{4}$ $\mathrm{Pan} \mathrm{Yu}^{3}$

'Third Department of Medical Oncology, Shannxi Provincial Cancer Hospital, ${ }^{2}$ Breast Surgery Center, Shannxi Provincial Cancer Hospital, Xi'an, ${ }^{3}$ Marketing Department, Novogene Bioinformatics Institute,

Beijing, ${ }^{4}$ Pathology Department, Shannxi Provincial Cancer Hospital, Xi'an, People's Republic of China

\section{Video abstract}

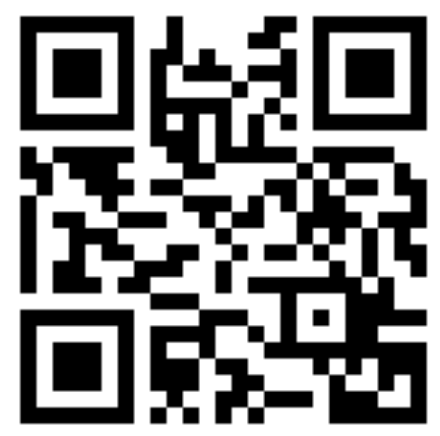

Point your SmartPhone at the code above. If you have a QR code reader the video abstract will appear. Or use: http://youtu.be/P0idArkplfs

Correspondence: Zheng Zhao Third Department of Medical Oncology, Shannxi Provincial Cancer Hospital, 309 Yanta West Road, Xi'an, Shanxi, 71006I, People's Republic of China

Email zhaozheng1513@163.com

Pan Yu

Novogene Bioinformatics Institute, 38 Xueqing Road, Haidian District, Beijing, 100083, People's Republic of China

Email yupan@novogene.com
Abstract: ROS1 fusion is a common genetic alteration in non-small-cell lung cancer. Crizotinib, an anaplastic lymphoma kinase inhibitor, shows efficacy in the treatment of lung cancer cases with $R O S 1$ translocation. We report the response to crizotinib of a lung adenocarcinoma patient harboring a novel SLC34A2-ROS1 fusion variant, which was different from the two common $S L C 34 A 2-R O S 1$ fusion types reported in the literature. After crizotinib administration, overall recovery was good in this patient; the primary lesion was successfully treated, the lymph node metastases had disappeared, and the metabolism was normal.

Keywords: SLC34A2-ROS1 fusion, crizotinib, lung adenocarcinoma, next-generation sequencing

\section{Introduction}

ROS1 fusion, a common genetic alteration in non-small-cell lung cancer (NSCLC), ${ }^{1}$ is present in about $2.4 \%$ of lung adenocarcinoma cases. Several fusion types have been reported, including CD74, SLC34A2, EZR, LRIG3, SDC4, TPM3, FIG GOPC, CCDC6, KDEL R2 LIMA1, MSN, CLTC, TMEM106B, and TPD52L1. ${ }^{2-5}$ The application of reliable screening methods for gene rearrangement detection is key in identifying patients suitable for tumor-targeted therapy., 5 To date, ROS1 fusions in NSCLC are most commonly diagnosed by florescence in situ hybridization (FISH) or amplification refractory mutation system-polymerase chain reaction (ARMS-PCR) ${ }^{6-10}$ However, ARMS-PCR and FISH are costly, less precise, and not suitable for detecting unknown significant mutations. ${ }^{11-16}$ Next-generation sequencing (NGS) is an optional method for identifying unknown genes with potential benefit. ${ }^{10,17-19}$ Crizotinib, a tyrosine kinase inhibitor targeting ALK and MET, was approved by the US Food and Drug Administration (FDA) on March 11, 2016, for the treatment of advanced metastatic ROS1-rearranged NSCLC. This report describes a patient successfully treated with crizotinib, in whom a novel SLC34A2-ROS1 fusion variant was identified. Written informed consent was obtained from the patient for the publication of this case report and any accompanying images. Approval from an ethics committee was not required due to this being a retrospective study and the information regarding the case being anonymous.

\section{Case report}

A 48-year-old female patient with back pain was admitted to the Shanxi Provincial Cancer Hospital; she was suspected with left breast cancer. Subsequently, lymph node 
metastasis of lung adenocarcinoma was considered in both sides of the neck and the left side of axillary lymph nodes after biopsy. She had 10 years of smoking history (1 pack/d) and no family history of genetic diseases. Biopsy samples were assessed for EGFR, ALK, and ROS1 gene mutations by ARMS-PCR (Amoy Diagnostics Co., Ltd, Xiamen, People's Republic of China); however, all were negative. The patient received 2 cycles of chemotherapy (docetaxel $240 \mathrm{mg}+$ cisplatin $240 \mathrm{mg}$ ). Because of progressive disease (PD), the treatment was discontinued on December 9, 2015. Positron emission tomography (PET)/computed tomographic (CT) imaging revealed that the tumor had developed multiple lymph node metastases. The chemotherapy regimen was replaced by 2 cycles of chemotherapy (pemetrexed $800 \mathrm{mg}$, intravenous [IV], d1; cisplatinum $20 \mathrm{mg} \mathrm{IV}, \mathrm{d} 1-5$, bevacizumab $0.5 \mathrm{IV} \mathrm{d} 1)$ and a combined 1 cycle of local radiotherapy $(4,400 \mathrm{cGy} / 22 \mathrm{f} / 5 \mathrm{w})$. CT assessment showed stable disease in the patient, and palliative treatment was performed by pemetrexed plus cisplatin chemotherapy. However, the chemotherapeutic treatment was discontinued because of severe bone marrow suppression.

With no effective treatment at hand, biopsy samples were assessed using the NGS-483 gene panel (Novogene Bioinformatics Technology Co., Ltd, Beijing, People's Republic of China). Biotinylated oligoprobes were designed along the norepeat regions of 483 genes containing all the exons and some introns. The amplified exonic and intronic DNA was then sequenced on HiSeq X Ten sequencer (Illumina, San Diego, CA, USA) producing paired 150 bp reads. In the 483 genes, NGS revealed a new ROS1 fusion (SLC34A2-ROS1) and the $T P 53$ p E132Q variation. Next, we assessed the causes of different detection results in the same samples. While analyzing ROS1 fusion sequence data, a novel SLC34A2-ROS1 fusion variant, different from the two common SLC34A2-ROS1 fusion types, was discovered (Figure 1). According to the sequencing data, the novel breakpoint was chr6:117653720, chr4:25678781. To verify the breakpoint, we respectively designed a primer pair based on the ROS1 gene's breakpoint downstream and left to the SLC34A2 gene's upstream breakpoint. Direct Sanger sequencing demonstrated that the $3^{\prime}$ untranslated region (3'UTR) of SLC34A2 in exon 13 was disrupted and inverted to connect a position of intronic_e32_e31 of ROS1 (Figure 2). The new breakpoint was not included in Catalogue Of Somatic Mutations In Cancer (COSMIC; http:// cancer.sanger.ac.uk/cosmic) and was located in the SLC34A2 gene of the last transcription region (extra exon 13, as the 3'UTR region, was not involved in translation). Therefore, conventional detection methods cannot be used.
On March 11, 2016, the FDA formally approved crizotinib (Pfizer) for the treatment of patients with advanced (metastatic) NSCLC harboring ROS1 gene fusion. Would the patient with the novel SLC34A2-ROS1 fusion variant showed no response to crizotinib? We predicted the protein domain of the new fusion gene, and the ROS1 tyrosine kinase domain could not be destroyed.

Combined with the above information, the female patient was started on treatment with crizotinib (250 mg, 2/d, d1-30) from March 24, 2016. After 2 weeks, the breast mass was reduced, and the patient reported relieved pain and improved quality of life. Two months later, according to PET/CT, most metastatic lymph nodes had disappeared, with the metabolism returning to normal. Eleven months later, the original irregular nodules in the basal segment of the right lung's lower lobe were slightly narrowed, with reduced metabolism; the shape was uniform, with rough edges and no nodules or pleural adhesion. The lesion increase, which reflects the primary malignant lesion (pneumotype of the lower lobe of the lung), was slightly smaller and more active after treatment. The anterior mediastinum septum, the left lung, the upper and lower sides of the collarbone, and the left armpit had lymph node metastases. The lymph nodes of interstitial lesions violated the left vertebra of the neck; after treatment, the metastatic lymph nodes disappeared, with the metabolism returning to normal. In this case, the external pressure of lymph nodes, the left armpit, the cervical lymph and blood vessels, left upper extremities, left breast area edema, and swelling were relieved after treatment, and remained so. No new tumor metastases were found in other organs and bones in other parts of the body. The patient's overall recovery was good; the primary lesion was relieved, the lymph node metastasis had disappeared, and the metabolism was normal (Figure 3).

\section{Discussion}

Crizotinib has been approved by FDA for the treatment of advanced metastatic NSCLC with ROS1 and $A L K$ rearrangements. ${ }^{1,7,10,17}$ In the Phase I clinical trial of crizotinib, 50 NSCLC patients with ROS1 fusion were enrolled, including 49 cases detected by FISH and 1 by reverse transcriptase-polymerase chain reaction (RT-PCR). The overall response rate (ORR) was $72 \%$, with a median duration of response (DOR) of 17.6 months and a median progressionfree survival (PFS) of 19.2 months. ${ }^{3}$

The SLC34A2-ROS1 fusion variant is a common ROS1 fusion in NSCLC accounting for the second highest percentage. ${ }^{4,5}$ It is likely generated from an intra-chromosomal 
A SLC34A2 gene

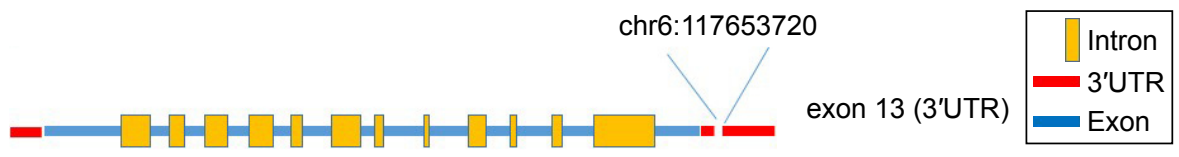

ROS1 gene

31 intron chr6:117653720
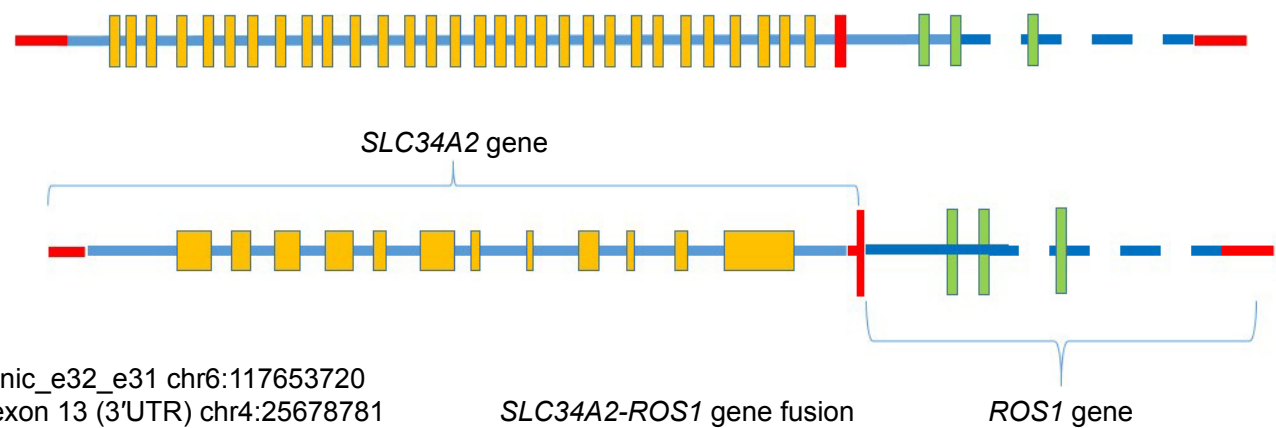

ROS1 intronic_e32_e31 chr6:117653720

SLC34A2 exon 13 (3'UTR) chr4.25678781 SLC34A2-ROS1 gene fusion

ROS1 gene

B

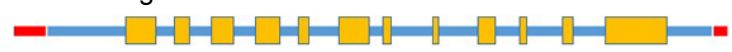

ROS1 gene
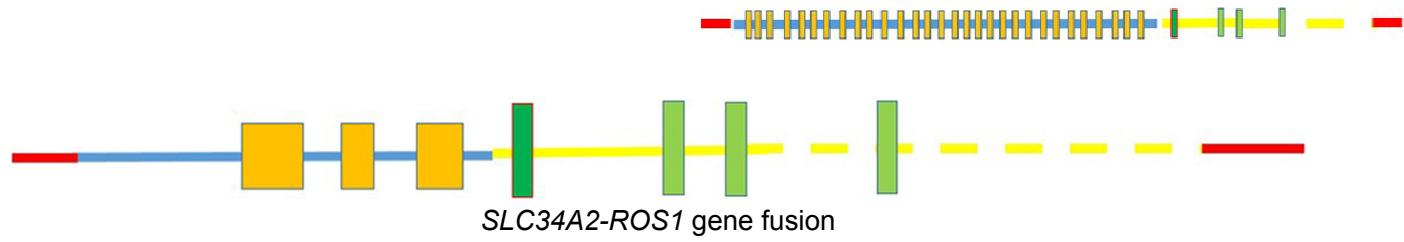

C

SLC34A2 gene

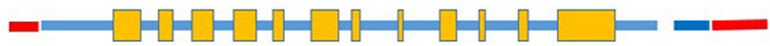

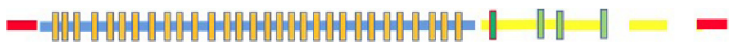
ROS1 gene

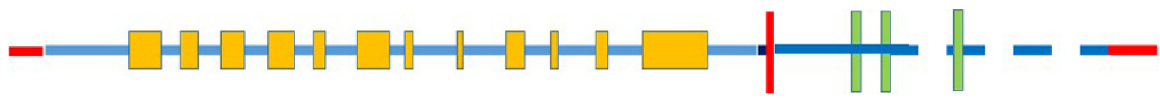

SLC34A2-ROS1 gene fusion

Figure I Structure of the novel SLC34A2-ROSI fusion gene.

Notes: $\mathbf{A}$ is the novel fusion gene and is different from the other two SLC34A2-ROSI fusion (B and C) variants. In the COSMIC database, two SLC34A2-ROSI fusion forms were found (B and C). In the novel SLC34A2-ROSI fusion, 3'UTR of SLC34A2 in exon 13 was disrupted and inverted to connect a position of intronic_e32_e3I of ROSI.

Abbreviations: COSMIC, Catalogue Of Somatic Mutations In Cancer; 3' UTR, 3’ untranslated region.

deletion and fusion, similar to $E M L 4-A L K$ generation in NSCLC. ${ }^{1,8,13,15}$ NGS fortunately revealed a new ROS1 fusion (SLC34A2-ROS1) and TP53 $\mathrm{p}$ E132Q variation; ALK fusion mutation was not found in this sample. The novel SLC34A2ROS1 fusion variant (3'UTR of SLC34A2 in exon 13 was disrupted and inverted to connect a position of intronic_e32_e31 of $R O S 1$ ) differed from the two previously discovered SLC34A2-ROS1 fusion types (Figure 1). Based on sequencing results, the novel breakpoint was chr6:117653720, chr4:25678781. After the administration of crizotinib for 2 weeks, the breast mass was reduced, pain was relieved, and the quality of life improved. After 2 months, PET/CT 

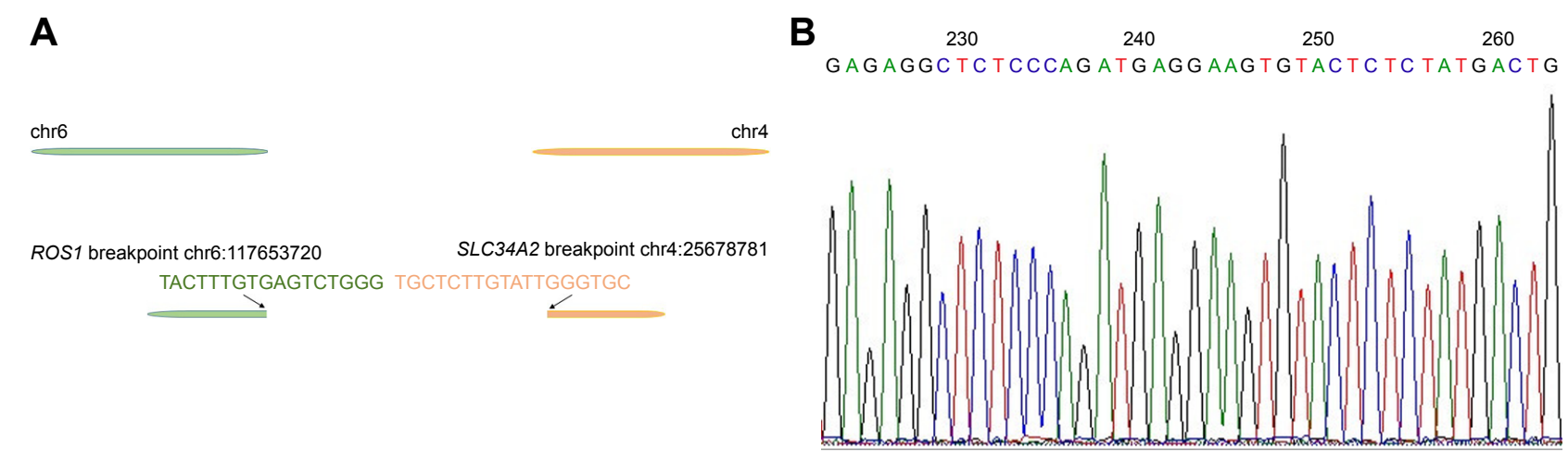

Figure 2 Accuracy verification of the novel SLC34A2-ROSI fusion by Sanger sequencing.

Notes: (A) Based on the results of the next-generation sequencing, we designed a primer pair based on the ROSI gene's breakpoint downstream and left to the SLC34A2 gene's upstream breakpoint; (B) the mutations in the sample DNA were sequenced by Sanger sequencing and the breakpoints in the next-generation sequencing are identical.

indicated a progressive relief; 11 months later, overall recovery was good in this patient, with primary lesion successfully treated, no lymph node metastases, and normal metabolism. The patient also harbored a TP53 $\mathrm{p} E 132 Q$ variation. Would this also explain why crizotinib was effective in this patient? The tumor suppressor gene TP53 is the most frequently mutated in NSCLC. It encodes a 393-aa protein with three distinct domains. TP53p E132Q exists on the binding domain of TP53; however, it may not affect the function of proteins. In addition, this remains unclear as no relevant literature was found. These findings suggest that the novel SLC34A2-ROS1 fusion shows good response to crizotinib. ARMS-PCR is a fast and sensitive method for evaluating the expression of known ROS1 fusion variants, ${ }^{14,16}$ for which specific primers have been designed. In a previous study, the sensitivity and specificity of ARMS-PCR for detecting ROS1 fusion were $100 \%$ and $85.1 \%$, respectively. ${ }^{10}$ There are two possible reasons why we failed to detect the ROSI fusion in the patient's sample: 1) only a small population of cancer cells might be carrying the ROS1 fusion and 2) this fusion might occur at the transcriptional level, with no translation. In this report, the rare $R O S 1$ variants were not detected by ARMS-PCR, since ARMS-PCR is based on cDNA after mRNA transcription. The new breakpoint was not included in COSMIC and was located in the last transcription region of the SLC34A2 gene; therefore, it could not be detected by ARMS-PCR. In the 483 genes, NGS revealed the new ROS1 fusion (SLC34A2$R O S 1)$; Direct Sanger sequencing demonstrated that the $3^{\prime} U T R$ of SLC34A2 in exon 13 was disrupted and inverted to connect a position of intronic_e32_e31 of ROS1. For further confirmation, a FISH assay (Guangzhou Anpingping [LBP] Pharmaceutical Technology Co., Ltd) was performed to check for this fusion variant; however, the results were negative. Also, we analyzed the reasons for the difference of detection result between the two methods. The gene fusion process is very complex; it is possible that in the upstream area ROS1
A

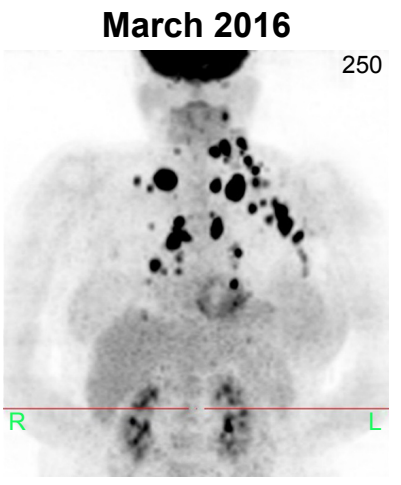

B

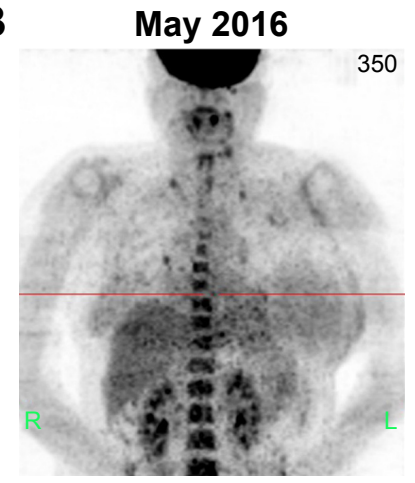

C February 2017

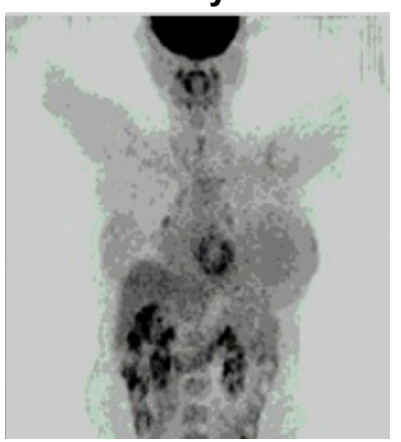

Figure 3 Computed tomography and positron emission tomography images of the right lower lobe.

Notes: Mediastinum, upper and lower bilateral clavicle area, left armpit, and lung lymph node metastases at treatment initiation (March 20I6; A). After treatment with crizotinib for 2 months (May 20I6; B), the metastatic lymph nodes had disappeared, with the metabolism returning to normal; II months later (February 20I7; C). No new tumor metastases were found in other organs and bones in other parts of the body. The patient's overall recovery was good; the primary lesion was relieved, the lymph node metastasis had disappeared, and the metabolism was normal. 
missing part of the area, by parts of SLC $34 A 2$ gene insert this part again, without affecting the other areas in the FISH. The red probe can also be combined with a green probe, which is still negative when examined. A study shows that the same phenomenon exists in the $A L K$ fusion. ${ }^{20}$ There is a complete possibility of a rearrangement is no separation of the red and green probes. NGS is an optional method for the detection of all forms of gene variation. Thus, the multi-platform technology of detection can maximally prolong patient survival. To monitor the expression of this fusion from diagnosis throughout the treatment, liquid biopsy-circulating tumor DNA was used with the NGS 483 gene panel. Circulating tumor DNA samples of the patient, in July 2016 and January 2017, respectively, were assessed by the NGS 483 gene panel; interestingly, the SLC34A2-ROS1 fusion variant had disappeared, in agreement with the PET/CT data.

\section{Disclosure}

Xuwei Wang and Pan Yu are employees of Novogene Bioinformatics Institute. The authors report no other conflicts of interest in this work.

\section{References}

1. Takeuchi K, Soda M, Togashi Y, et al. RET, ROS1 and ALK fusions in lung cancer. Nat Med. 2012;18(3):378-381.

2. Bergethon $\mathrm{K}$, Shaw AT, Ou SHI, et al. ROS1 rearrangements define a unique molecular class of lung cancers. J Clin Oncol. 2012;30(8):863-870.

3. Shaw AT, Ou SH, Bang YJ, et al. Crizotinib in ROS1-rearranged nonsmall-cell lung cancer. N Engl J Med. 1971;372(7):1963-1971.

4. Awad MM, Katayama R, Mctigue M, et al. Acquired resistance to crizotinib from a mutation in CD74-ROS1. N Engl J Med. 2013; 368(25):2395-2401.

5. Rimkunas VM, Crosby KE, Li D, et al. Analysis of receptor tyrosine kinase ROS1-positive tumors in non-small cell lung cancer: identification of a FIG-ROS1 fusion. Clin Cancer Res. 2012;18(16):4449-4457.

6. Lee J, Lee SE, Kang SY, et al. Identification of ROS1 rearrangement in gastric adenocarcinoma. Cancer. 2013;119(9):1627-1635.
7. Yoshida A, Kohno T, Tsuta K, et al. ROS1-rearranged lung cancer: a clinicopathologic and molecular study of 15 surgical cases. Am J Surg Pathol. 2013;37(4):554-562.

8. Davies KD, Le AT, Theodoro MF, et al. Identifying and targeting ROS1 gene fusions in non-small cell lung cancer. Clin Cancer Res. 2012;18(17):4570-4579.

9. Yoshida A, Tsuta K, Wakai S, et al. Immunohistochemical detection of ROS1 is useful for identifying ROS1 rearrangements in lung cancers. Mod Pathol. 2014;27(5):711-720.

10. Shan L, Lian F, Guo L, et al. Detection of ROS1 gene rearrangement in lung adenocarcinoma: comparison of IHC, FISH and real-time RT-PCR. PLoS One. 2014;10(3):e0120422.

11. Suehara Y, Arcila M, Wang L, et al. Identification of KIF5B-RET and GOPC-ROS1 fusions in lung adenocarcinomas through a comprehensive mRNA-based screen for tyrosine kinase fusions. Clin Cancer Res. 2012;18(24):6599-6608.

12. Cai W, Li X, Su C, et al. ROS1 fusions in Chinese patients with nonsmall-cell lung cancer. Ann Oncol. 2013;24(7):1822-1827.

13. Peraldo NC, Cavalloni G, Balsamo A, et al. Screening for the FIG-ROS1 fusion in biliary tract carcinomas by nested PCR. Genes Chromosomes Cancer. 2014;53(12):1033-1040.

14. Pikor LA, Ramnarine VR, Lam S, Lam WL. Genetic alterations defining NSCLC subtypes and their therapeutic implications. Lung Cancer. 2013;82(2):179-189.

15. Yamaguchi N, Lucenaaraujo AR, Nakayama S, et al. Dual ALK and EGFR inhibition targets a mechanism of acquired resistance to the tyrosine kinase inhibitor crizotinib in ALK rearranged lung cancer. Lung Cancer. 2014;83(1):37-43.

16. Pan Y, Zhang Y, Li Y, et al. ALK, ROS1 and RET fusions in 1139 lung adenocarcinomas: a comprehensive study of common and fusion pattern-specific clinicopathologic, histologic and cytologic features. Lung Cancer. 2014;84(2):121-126.

17. Lukas RV, Hasan Y, Nicholas MK, Salgia R. ROS1 rearranged nonsmall cell lung cancer brain metastases respond to low dose radiotherapy. J Clin Neurosci. 2015;22(12):1978-1979.

18. Le X, Freed JA, Vanderlaan PA, et al. Detection of crizotinib-sensitive lung adenocarcinomas with MET, ALK, and ROS1 genomic alterations via comprehensive genomic profiling. Clin Lung Cancer. 2015;16(5):e105-e109.

19. Boyle TA, Masago K, Ellison KE, Yatabe Y, Hirsch FR. ROS1 immunohistochemistry among major genotypes of non-small-cell lung cancer. Clin Lung Cancer. 2015;16(2):106-111.

20. Ali SM, Hensing T, Schrock AB, et al. Comprehensive genomic profiling identifies a subset of crizotinib-responsive ALK-rearranged nonsmall cell lung cancer not detected by fluorescence in situ hybridization. Oncologist. 2016;21(6):762-770.
OncoTargets and Therapy

\section{Publish your work in this journal}

OncoTargets and Therapy is an international, peer-reviewed, open access journal focusing on the pathological basis of all cancers, potential targets for therapy and treatment protocols employed to improve the management of cancer patients. The journal also focuses on the impact of management programs and new therapeutic agents and protocols on

\section{Dovepress}

patient perspectives such as quality of life, adherence and satisfaction. The manuscript management system is completely online and includes a very quick and fair peer-review system, which is all easy to use. Visit http://www.dovepress.com/testimonials.php to read real quotes from published authors. 